University of Nebraska - Lincoln

DigitalCommons@University of Nebraska - Lincoln

10-15-1995

\title{
Correlation between screening and electron effective mass across the nonmetal-metal transition in ultrathin films
}

Jiandi Zhang

University of Nebraska-Lincoln, jiandiz@lsu.edu

D.N. Mcllroy

University of Nebraska-Lincoln

Peter A. Dowben

University of Nebraska-Lincoln, pdowben@unl.edu

Follow this and additional works at: https://digitalcommons.unl.edu/physicsdowben

Part of the Physics Commons

Zhang, Jiandi; Mcllroy, D.N.; and Dowben, Peter A., "Correlation between screening and electron effective mass across the nonmetal-metal transition in ultrathin films" (1995). Peter Dowben Publications. 50.

https://digitalcommons.unl.edu/physicsdowben/50

This Article is brought to you for free and open access by the Research Papers in Physics and Astronomy at DigitalCommons@University of Nebraska - Lincoln. It has been accepted for inclusion in Peter Dowben Publications by an authorized administrator of DigitalCommons@University of Nebraska - Lincoln. 


\title{
Correlation between screening and electron effective mass across the nonmetal-metal transition in ultrathin films
}

\author{
Jiandi Zhang, D. N. McIlroy, and P. A. Dowben \\ Department of Physics and Astronomy and the Center for Materials Research and Analysis, Behlen Laboratory of Physics, \\ University of Nebraska-Lincoln, Lincoln, Nebraska 68588-0111
}

(Received 27 December 1994; revised manuscript received 27 June 1995)

\begin{abstract}
Angle-resolved photoemission and resonant photoemission have been used to determine the relative changes of the electron effective mass and the screening parameter across the nonmetal to metal transition in the magnesium thin films on $\mathbf{M o}(112)$. Both the screening length and the electron effective mass exhibit a gradual change with $\mathrm{Mg}$ coverage in the transition range between half and one monolayer. The relationship of these two different measures of metallicity is in surprisingly good agreement with the relationship predicted by theory for the Mott-Hubbard transition. Our results indicate the importance of electron-electron correlation within the overlayer.
\end{abstract}

\section{INTRODUCTION}

There is now considerable experimental and theoretical evidence indicating that ultrathin films of materials, usually considered to be metallic, exhibit nonmetallic behavior. $^{1-9}$ These systems undergo nonmetal-to-metal transitions with changing film density, atomic structure, or thickness. Despite that it is now generally accepted that a nonmetal-to-metal transition can occur on not only insulator and semiconductor surfaces but also metal substrates, a detailed understanding of the fundamental mechanisms continues to remain elusive. ${ }^{1}$

The models associated with the metal-insulator transition were developed for bulk materials. These models include the Mott-Hubbard transition based on the electron correlation, the Wilson transition based on the electron orbital hybridization, and the Peierls transition attributable to the lattice distortions. ${ }^{1}$ For divalent atoms adsorbed on metal surfaces, ${ }^{3-7}$ photoemission, resonant photoemission, and inelastic electron scattering experiments reveal a nonmetal-metal transition with increasing coverage. The evolution in metallicity of these divalent metal films cannot be usually understood by a simple one-particle picture such as a Wilson-like transition model. The transition seems to be gradual, not abrupt, ${ }^{7}$ and the details of electronic structure do not correspond to the nonmetallic phase expected for a Wilson transition. ${ }^{4}$

Experimentally, the nonmetal-to-metal transition can be reflected by the changes in the valence electronic structure $^{2-9}$ and the electron screening effects in many-body processes in photoemission. ${ }^{3-5,7}$ Changes in the electron effective mass and the dielectric response (which is related to the screening length or screening parameter) can be anticipated across the metal-nonmetal transition. For a highly correlated electron system close to the metalnonmetal transition, a strong enhancement in electron effective mass due to the significant electron-electron interaction is anticipated. ${ }^{10-15}$ Such an enhancement in the effective mass is even stronger in a two-dimensional electron system than that expected in a three-dimensional system. ${ }^{14,16}$ Recently, Tokura et al. ${ }^{17}$ have found that the effective mass, spin susceptibility, and specific heat show a strong enhancement across the metal-nonmetal transition in the compound of $\mathrm{Sr}_{1-x} \mathrm{La}_{x} \mathrm{TiO}_{x}$ and the enhancement is attributable to the electron-correlation effects. There are, as yet, no corresponding experimental results for a pure metal overlayer and no evidence that for a reduced dimensional system the effective mass can be experimentally related to screening. The main aim of this paper is to provide a connection between the change of electron in-plane effective mass and the evolution of the screening parameter across the nonmetal-metal transition in the $\mathrm{Mg}$ thin films with changing coverage.

\section{EXPERIMENT}

The experiments were conducted on a 6-m toroidal grating monochromator beam line at the Synchrotron Radiation Center in Stoughton, Wisconsin. Angleresolved photoemission was conducted in a UHV chamber as described in our previous effort ${ }^{7}$ on this system. The constant initial state (CIS) spectra were taken by scanning the photon energy and electron kinetic energy simultaneously to record the intensity changes of the same initial state. ${ }^{7}$

Magnesium was deposited at room temperature and coverages were determined using a quartz crystal thinfilm thickness monitor, core-level intensities, and lowenergy electron diffraction (LEED). For submonolayer coverages, we found LEED to be dominated by the $1 \times 2$ and $1 \times 1$ diffraction patterns, characteristic of half and one monolayer (nominal coverage) structures, respective$1 y$, in spite of suggestions of a number of complicated submonolayer phases for this system. ${ }^{18}$

\section{EVOLUTION OF ELECTRONIC STRUCTURE}

As discussed in previous work, ${ }^{7}$ there are two $\mathrm{Mg}$ induced features with binding energies of $0.5 \pm 0.1$ and $1.6 \pm 0.1 \mathrm{eV}$ near the Fermi level that evolve with increas- 
ing $\mathrm{Mg}$ coverages on the $\mathrm{Mo}(112)$ surface. These states are characteristic of the $\mathrm{Mg}$-induced electronic structure, as described elsewhere. ${ }^{7}$ The state with a binding energy of $1.6 \pm 0.1 \mathrm{eV}$ at normal emission decreases in binding energy to $1.45 \pm 0.1 \mathrm{eV}$ at completion of two monolayers of coverage. This state has been observed for the $\mathrm{Mg}(0001)$ surface ${ }^{19}$ and is located in the $\Gamma_{3}^{+}-\Gamma_{4}^{-}$bulk band gap as a surface state. Similar surface states have been observed for other alkaline-earth metal surfaces. ${ }^{20}$ The other $\mathrm{Mg}$-induced state at a binding energy $0.5 \pm 0.1$ $\mathrm{eV}$ appears at about half of a monolayer and becomes well resolved following the completion of the first monolayer of $\mathrm{Mg}$. Between 0.5 and $1.0 \mathrm{ML}$, there is an increase in the density of states at $E_{F}$, as described in previous work. ${ }^{7}$ This change corresponds to the nonmetal-tometal transition in the $\mathrm{Mg}$ thin films. ${ }^{6,7}$ At large coverages, a feature at $5.7-\mathrm{eV}$ binding energy is induced by the Mg overlayer ${ }^{7}$ and develops into the bulk $\mathrm{Mg}$ band. ${ }^{19}$ While this band appears to be in superficial agreement with theoretical efforts for a $\mathrm{Mg}$ monolayer, ${ }^{21}$ the band is in fact a bulk band.

In order to further illustrate details of the evolution of the electronic structure in the overlayers with increasing Mg coverage, angle-resolved photoemission spectra have been taken to map out the band structure with increasing $\mathrm{Mg}$ coverage. The original spectra characteristic of $0.5-$, 1.0-, 1.5-, and 2.0-ML magnesium films on Mo(112) are shown in Fig. 1. The evolution of the valence electronic structure of the $\mathrm{Mg}$ overlayers as a function of thickness has been characterized along the two principal symmetry directions of the surface Brillouin zone (SBZ). The contribution from the substrate features can be subtracted since the band structure of the Mo(112) surface is different from low coverage of the overlayer (as shown in Fig. 2) and discussed elsewhere. ${ }^{22}$ The dispersion of the $\mathrm{Mg}$ state at about $1.6 \mathrm{eV}$ (see Fig. 1) has been plotted along the $\bar{\Gamma}-\bar{X}$ and $\bar{\Gamma}-\bar{Y}$ symmetry directions of the SBZ for different coverages, as shown in Fig. 3. The dispersion of the $\mathrm{Mg}$ state at a binding energy of $0.5 \mathrm{eV}$ along the $\bar{\Gamma}-\bar{X}$ symmetry direction is also plotted in Fig. 3 . The band structure of the $\mathrm{Mg}$ overlayers for more coverages than those shown in Fig. 3 have also been determined. These results are consistent with the gradual evolution towards a highly dispersive band structure for the high coverage metallic $\mathrm{Mg}$ overlayer, as indicated in Fig. 3. For very thick films, the experimental band structure qualitatively agrees with theoretical results ${ }^{21}$ though the data differ from the calculated binding energies.

Among the interesting results obtained from this investigation are the changes in the dispersion of these two Mg-induced states with film thickness. First, we note that there is an anisotropy of the dispersion along the $\bar{\Gamma}$ $\bar{X}$ versus $\bar{\Gamma}-\bar{Y}$ direction (as seen in both Fig. 1 and Fig. 3 ). Along the $\bar{\Gamma}-\bar{X}$ direction, these states exhibit dramatic but gradual changes in their dispersions and eventually disperse across $E_{F}$ for high $\mathrm{Mg}$ coverages. Along the $\vec{\Gamma}$ $\bar{Y}$ direction, there is almost no change in dispersion or band structure with increasing $\mathrm{Mg}$ coverage.

The low-binding-energy $\mathrm{Mg}$ state (at $0.5 \mathrm{eV}$ ) exhibits a different change in the dispersion behavior as a function of coverage as compared to that of the $1.6-\mathrm{eV}$ state (about
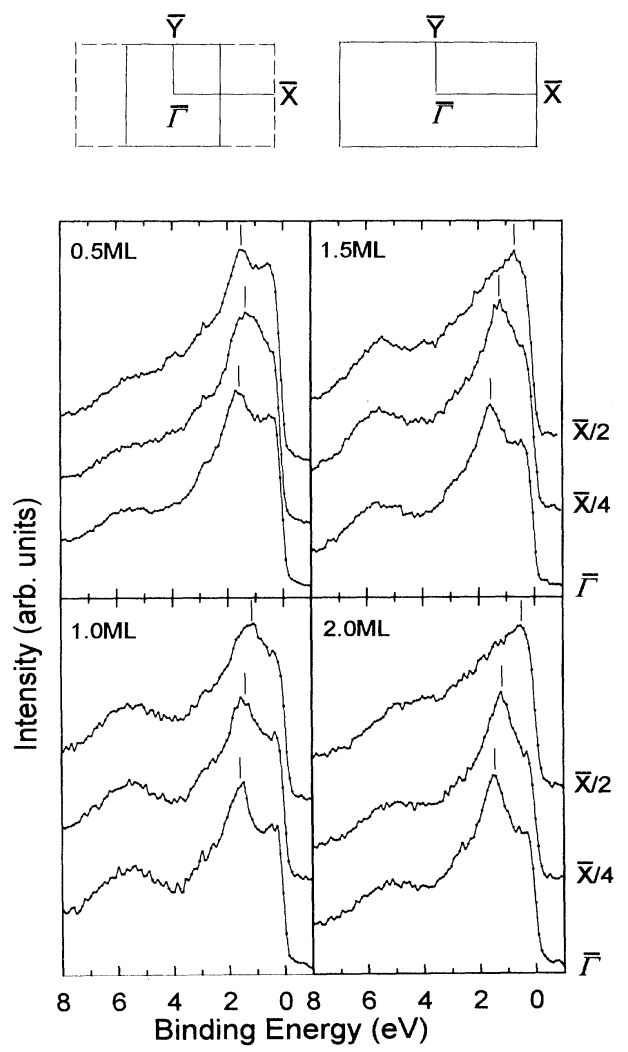

FIG. 1. Angle-resolved photoemission spectra of $\mathrm{Mg} / \mathrm{Mo}(112)$ along the $\bar{\Gamma}-\bar{X}$ direction with light of $70-\mathrm{eV}$ photon energy at an incidence angle of $70^{\circ}$. The surface Brillouin zones of $\mathrm{Mg}$ overlayers corresponding to $p(1 \times 2)$ and $p(1 \times 1)$ (solid) and the clean Mo(112) surface (dashed) are shown in the top.

$\bar{\Gamma}$ ). It should be noted that the binding energy of the $0.5-\mathrm{eV}$ feature is subject to substantial error due to the Fermi function (possibly resulting in an apparent increase in binding energy). Nonetheless, our results clearly point toward several conclusions. In the coverage range between half and one monolayer, the state at about $0.5-\mathrm{eV}$

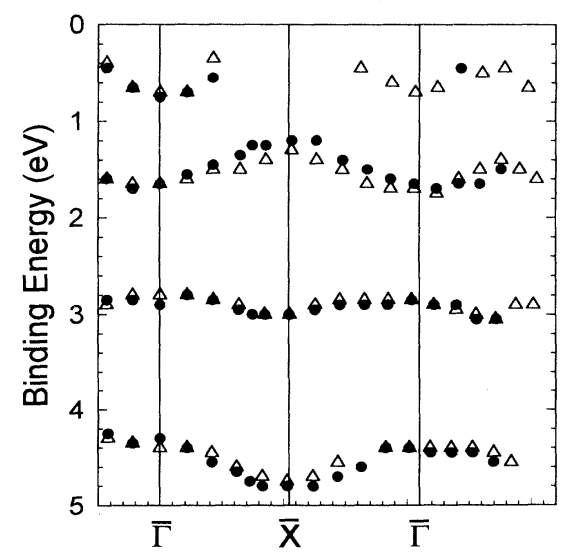

FIG. 2. The measured band structure of the Mo(112) surface along the $\bar{\Gamma}-\bar{X}$ direction. The data were taken using $70-\mathrm{eV} p-$ $(O)$ and $s-(\triangle)$ polarized light. 

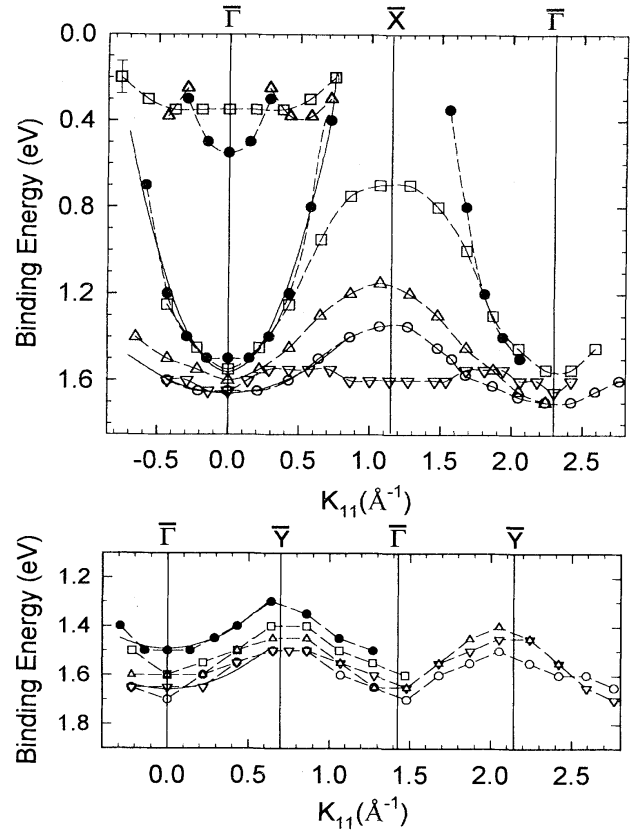

FIG. 3. The measured dispersion of the Mg-induced bands along both the $\bar{\Gamma}-\bar{X}$ (top) and $\bar{\Gamma}-\bar{Y}$ (bottom) directions, at different coverages $[0.4 \mathrm{ML}(\nabla), 0.6 \mathrm{ML}(\bigcirc), 0.7 \mathrm{ML}(\triangle), 1$ ML ( $\square$ ), and $2 \mathrm{ML}(O)$ ]. The dispersions in the first surface Brillouin zone have been fit with a parabolic function and correspond to the solid curves; the dashed lines are only for guidance. Light with $70-\mathrm{eV}$ photon energy and $70^{\circ}$ incidence angle was used.

binding energy only appears below $E_{F}$ away from normal emission, although this state does disperse across $E_{F}$, as seen for the 0.7-ML overlayer (Figs. 1 and 3). For submonolayer coverages, the dispersion of this band is consistent with the surface Brillouin zone imposed by a $p(1 \times 2)$ structure. Above a coverage of one monolayer, this state appears with largest binding energy at $\bar{\Gamma}$ and disperses parabolically across the $E_{F}$, as does the $1.6-\mathrm{eV}$ feature. Both the 0.5- and 1.6-eV states are present for coverages above half a monolayer. The existence of these states in the submonolayer regime suggests that there are two domains (coexistence of phases) in the overlayer structure. Since there are only two valence electrons per $\mathrm{Mg}$ atom, one band may correspond to the $p(1 \times 2)$ structure and the other to the $p(1 \times 1)$ structure.

Such a dramatic change in the band structure can also be accompanied by a surface-structure phase transition. When adsorbed on corrugated surfaces of transition metals such as $\mathrm{W}(112)$ and $\mathrm{Mo}(112)$, many alkali and alkaline-earth adsorbates form linear chains that are oriented perpendicular to the furrow direction at low coverages. At higher coverages, continuous compression along the surface furrow direction occurs, finally resulting in the $p(1 \times 1)$ structure at the completion of one monolayer. ${ }^{6,18}$ From our LEED and core-level measurements, Mg overlayers form a $p(1 \times 2)$ structure on the Mo(112) surface at a coverage of $0.5 \mathrm{ML}$, with a lattice spacing of $4.45 \AA$ perpendicular to the surface furrow direction and $5.46 \AA$ in the furrow direction.

The change in dispersion from a localized state to a free-electron-like band should be due to the increase of the hybridization of electron orbitals in the overlayer by decreasing the atomic distance with increasing coverage. This explains, at least qualitatively, the anisotropy in the dispersion between the two perpendicular directions $(\bar{\Gamma}-\bar{X}$ and $\bar{\Gamma}-\bar{Y})$. The small bandwidth along the $\bar{\Gamma}-\bar{Y}$ direction is due to the large atomic distance $(4.45 \AA)$ for a wide range of coverages. Along the $\bar{\Gamma}-\bar{X}$ direction, the compression within the overlayer reduces the atomic distance, which has a pronounced effect on the band dispersion. At low coverages (below $0.5 \mathrm{ML}$ ), the large atomic distances prevent the electron orbitals from hybridizing, thereby keeping the electrons in the overlayer localized.

As the coverage increases beyond a half monolayer, there is a possible commensurate-incommensurate transition $^{6}$ of the overlayer structure and the atomic distance along the substrate furrow shrinks to $2.73 \AA$ at the completion of the first monolayer. Obviously, the band dispersion qualitatively reflects the structural transitions and the metallization within the overlayer. The onset of substantial dispersion as well as the dispersion of the Mg-induced band across $E_{F}$ clearly indicate that there is a nonmetal-to-metal transition between a half and one monolayer of coverage, consistent with previous conclusions. ${ }^{6,7}$ At the very least, the data indicate that there is one metallic phase (of two possible coexisting phases) within the overlayer.

\section{CHANGE IN ELECTRON EFFECTIVE MASS}

By fitting to the dispersion of the 1.6-eV (at $\bar{\Gamma}$ ) feature with a parabolic function centered about $\bar{\Gamma}$, the effective mass of this band as a function of coverage has been determined. The results for both the $\bar{\Gamma}-\bar{X}$ and $\bar{\Gamma}-\bar{Y}$ directions are presented in Fig. 4. The effective mass of this

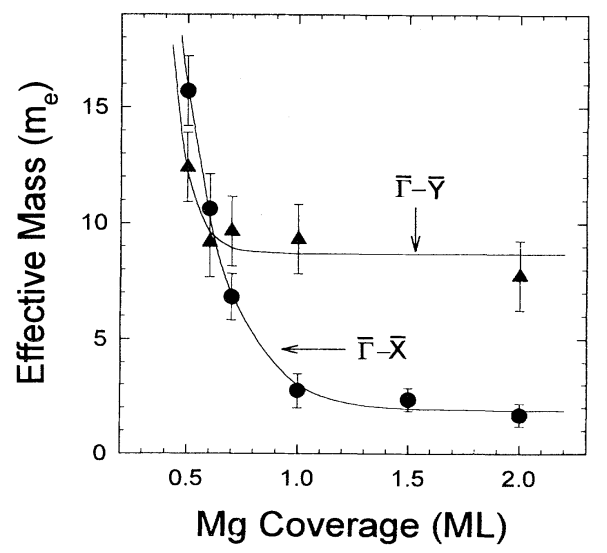

FIG. 4. The in-plane effective mass of the 1.6-eV (at $\bar{\Gamma}$ ) $\mathrm{Mg}$ state along the $\bar{\Gamma}-\bar{X}(0)$ and $\bar{\Gamma}-\bar{Y}(\boldsymbol{\Delta})$ directions, as a function of $\mathrm{Mg}$ coverage. The solid curves are the results of fits of the experimental data with an exponential function, and are meant only to be a guide. 
state changes substantially with coverage in the $\bar{\Gamma}-\bar{X}$ direction. The in-plane effective mass of this state along the $\bar{\Gamma}-\bar{X}$ decreases dramatically with increasing film thickness from $m^{*} / m_{e}=15.7$ at $0.5 \mathrm{ML}$ to 1.68 at $2 \mathrm{ML}$. The largest decrease in the effective mass takes place in the coverage range between a half and one monolayer. In contrast, the change in the effective mass along the $\bar{\Gamma}-\bar{Y}$ direction is quite small and this change occurs mostly around half a monolayer.

In general local-density approximation theory, the effective mass can be expressed as

$$
m^{*} / m_{e} \propto\left[1+\partial \Sigma(k, \varepsilon) / \partial \varepsilon_{k}\right]^{-1},
$$

where $\Sigma(k, \varepsilon)$ is the electron self-energy function involving many-body effects. ${ }^{23}$ In ordinary metals (near-freeelectron systems), the contribution to the effective mass from the electron-electron interaction is small. On the other hand, according to the Gutzwiller approach to the Hubbard model, ${ }^{24}$ there is not only a transition from a localized to a delocalized state expected in a strongly correlated system, but the effective mass and the screening length, as well as the static spin susceptibility, are found to be divergent when the system exhibits a Mott-insulator phase. ${ }^{10-12}$ The change of the effective mass is found to be related to the change of the electron-correlation energy (due to many-body effects). ${ }^{10-16}$ As can be seen in Fig. 4, the gradual decrease of the effective mass implies a gradual change in the electron-electron interaction instead of a discontinuous change as expected from simple models. Yet, this change causes a transition from a bondlike (localized) to a bandlike (delocalized) electronic phase, i.e., a nonmetal-to-metal transition.

The nonmetal-to-metal transition for $\mathbf{M g}$ on $\mathrm{Mo}(112)$ is a gradual transition (instead of an abrupt transition as expected for the Wilson-type transition), as is evidenced by the gradual evolution of the band structure (this work and previous results ${ }^{7}$ ). As we postulate that there are domains of two overlayer structures in the coverage range between a half and one monolayer, the overall effects of the two domains on the electronic structure from both domains provide a continuous evolution in metallicity even though there may exist an abrupt change for a perfect single domain structure within the overlayer. The gradual nonmetal-to-metal transition for the $\mathrm{Mg}$ two-dimensional overlayer that we observe is similar to that predicted for Mg clusters by Gong, Zheng, and $\mathrm{He}^{25}$ In the case of $\mathrm{Mg}$ clusters, theoretical calculations for small clusters show that there is an energy gap as high as $2.3 \mathrm{eV}$. With increasing cluster size this "band" gap gradually narrows and finally closes at a cluster size of 33 atoms. As with mercury overlayers, ${ }^{3-5}$ there is a surprising similarity between the development of electronic structure with increasing film thickness and increasing cluster size of free clusters.

Stronger electron-correlation effects in a twodimensional layer than that in bulk system are observed for Mg. This is clearly seen by comparing the effective mass in metallic $\mathrm{Mg}$ layers on $\mathrm{Mo}(112)$ with that of the $\mathrm{Mg}(0001)$ surface. For $\mathbf{M g}(0001)$, the corresponding state $(1.7 \mathrm{eV}$ at $\bar{\Gamma})$ disperses toward $E_{F}$ when probed away from the zone center and exhibits a parabolic freeelectron-like dispersion with an effective mass $m^{*} / m_{e}$ of 1.04. ${ }^{19}$ Even for metallic Mg overlayers ( $\geq 2 \mathrm{ML}$ thickness), the effective mass of the $1.6-\mathrm{eV}$ band is still larger than that of the corresponding band of the $\mathrm{Mg}(0001)$ surface (1.68 compared to 1.04 ). This is not surprising since the influence of many-body effects on the quasiparticle band structure should be larger in two dimensions than in three, as shown both theoretically ${ }^{14,15,26}$ and experimentally. 27

\section{THE CORRELATION BETWEEN THE SCREENING AND EFFECTIVE MASS}

We seek to find the connection between the effective mass of electrons within the $\mathrm{Mg}$ overlayer and the screening parameter. Increasing metallicity will increase the density of free electrons so as to increase the screening effects within the overlayer. By application of ThomasFermi screening theory to a two-dimensional system, ${ }^{5,28-33}$ a relationship between the screening parameter $l_{s}$ (which is the inverse of the screening length) and the density of free electrons $n_{s}$ can be obtained as

$$
\kappa l_{s}=\left(2 \pi e^{2} / k_{B}\right) n_{s} T,
$$

where $\kappa$ is the dielectric constant and $T$ the temperature of the system. Furthermore, increasing metallicity reduces the localization and effective mass of the electrons in the overlayer as well as strongly affecting the screening. A quantitative relationship between the effective mass and the screening parameter (or the screening length) should give an indication of the nature of the metallization of the overlayers.

A relative change in the screening effects across the nonmetal-to-metal transition in a thin film can be measured by resonant photoemission. ${ }^{3-5}$ In resonant photoemission like CIS, a core electron is excited to an unoccupied state near the $E_{F}$, forming a transient excited state or exciton. The decay of the exciton in the final state is identical to the direct photoemission process and drives a resonant enhancement of valence-band photoemission features at the characteristic photon energy (i.e., the resonance photon energy). The formation and decay of a core exciton in the resonant photoemission process can be affected by the screening. ${ }^{4}$ Such effects are reflected by the changes of resonance photon energy, intensity, and half-width. ${ }^{4,5}$ Generally, increased screening (due to increasing metallicity of the overlayer, in our case by increasing the $\mathrm{Mg}$ coverage) will cause a decrease in the resonance photoemission intensity and an increase in both resonance photon energy and half-width. ${ }^{4}$ For $\mathbf{M g}$ overlayers on a Mo(112) surface, dramatic changes in intensity and position (i.e., the resonance photon energy) of Mg $2 p \rightarrow \varepsilon d$ shape resonance have been observed with increasing $\mathrm{Mg}$ coverage. ${ }^{7}$ This is consistent with the results obtained for the evolution of the band structure with increasing magnesium coverage. The resonant photoemission also indicates that there is a gradual nonmetal-tometal transition in the $\mathrm{Mg}$ overlayers in the coverage range between 0.5 and $1.0 \mathrm{ML} .^{7}$ 
To obtain the correlation between the effective mass and the screening across the nonmetal-metal transition, the effective mass of the electrons in the $\mathrm{Mg}$-induced state with the binding energy of $1.6 \mathrm{eV}$ and the intensity of $\mathrm{Mg} 2 p \rightarrow \varepsilon d$ shape resonance have been plotted as a function of $\mathrm{Mg}$ coverage in Fig. 5. Dramatic decreases of both the effective mass and the resonance intensity can be seen in the nonmetal-to-metal transition range between half and one monolayer. In order to obtain quantitative relations between these two physical quantities and the Mg coverage, the decay of both the effective mass and the resonance intensity with coverage has been fitted to an exponential function of coverage for the transition range 0.5-1.0 ML, and can be expressed as

$$
\begin{aligned}
& m^{*}(\theta) / m_{e}=a+b \exp (-5.02 \theta), \\
& I(\theta) / I_{b}=c+d \exp (-2.49 \theta),
\end{aligned}
$$

where $m_{e}$ is the free-electron mass, $I_{b}$ the resonance intensity for the fully metallic (bulklike) layer, and $\theta$ the $\mathrm{Mg}$ coverage. One of the most important results is that the exponential decay parameter for the effective mass (with fitting value of 5.02) is about twice that of the resonance intensity (with fitting value of 2.49 ), so that there exists an experimental scaling relationship:

$$
m^{*}(\theta) / m_{e} \propto\left\{I(\theta) / I_{b}\right\}^{2}
$$

across the nonmetal-to-metal transition in the overlayers. Generally, the resonance intensity is inversely proportional to the screening parameter (which is the inverse of the screening length), ${ }^{5}$ i.e.,

$$
\left.I(\theta)\right|_{\text {resonance }} \propto 1 / l_{s}(\theta),
$$

where $l_{s}$ is the screening parameter of the overlayer. According to Eqs. (4) and (5), the empirical relationship characterizing the nonmetal-to-metal transition in the $\mathrm{Mg}$ overlayers can be expressed as

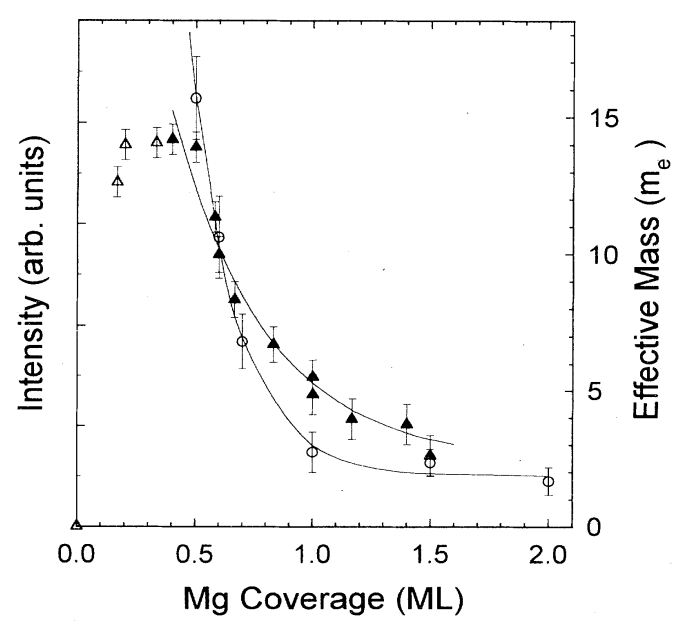

FIG. 5. The in-plane effective mass of the $\mathrm{Mg}$-induced state at $1.6 \mathrm{eV}($ at $\bar{\Gamma})$ along the $\bar{\Gamma}-\bar{X}(O)$ and the intensity $(\Delta)$ of $\mathrm{Mg}$ $2 p \rightarrow \varepsilon d$ shape resonance as a function of $\mathrm{Mg}$ coverage.

$$
m^{*}(\theta) / m_{e} \propto\left\{l_{s}(\theta) / l_{b}\right\}^{-2},
$$

where $l_{b}$ is the screening parameter when the overlayer is bulklike and fully metallic. This result is exactly the same as that predicted by Brinkman and Rice ${ }^{10-12}$ based on the Mott-Hubbard model. According to Gutzwiller's variational approach as applied to the Hubbard model, ${ }^{24}$ both the effective mass of electrons and the screening length diverge at a critical value of the intra-atomic Coulomb interaction $U_{C}$, which corresponds to a metalinsulator transition. In the metallic phase and near the transition, there exists the following scaling relationship:

$$
q_{s}^{2} \propto 1-\left(U / U_{C}\right)^{2} \propto\left(m^{*} / m_{e}\right)^{-1}
$$

as $U \rightarrow U_{C}$, where $q_{s}$ is the screening length $\left(q_{s} \equiv 1 / l_{s}\right)$ and $U$ is the intra-atomic Coulomb interaction in the Hubbard model. The Hamiltonian of the system is simply expressed as

$$
H=\sum_{(i, j, \sigma)} t_{i j} c_{i \sigma}^{+} c_{j \sigma}+U \sum_{(i)} n_{i \uparrow} n_{i \downarrow}
$$

where $n \equiv c^{+} c$, the occupation-number operator. The first part is the kinetic energy due to hopping between sites $i$ and $j$ and the second part is the Coulomb interaction energy. According to this model, $m^{*} \rightarrow m_{e}, U \rightarrow 0$, and $l_{s} \rightarrow l_{b} \rightarrow \infty$ in the free-electron-like metal system and $m^{*} \rightarrow \infty, U \rightarrow U_{C}$, and $l_{s} \rightarrow 0$ in the Mott-insulator phase.

As is well known, the metal-insulator transition predicted from the above theory was originally applied to a half-filled system (one electron per site, like the alkali metals). However, a recent theory based on the fundamental interplay between kinetic and Coulombic energies and the van der Waals interactions, proposed by Garcia and co-workers, ${ }^{34,35}$ has been successfully used to explain the transition from localized to delocalized electronic states in divalent metal clusters.

The good agreement between the empirical relationship derived for the $\mathbf{M g}$ overlayer system and that derived from the Mott-Hubbard model suggests that, even though a divalent metal system is not exactly the system originally described by the Hubbard model, this model may be helpful to understand the nonmetal-to-metal transition in our divalent layer system. It also suggests that unlike a simple one-particle picture (i.e., noninteracting electron model) like that in a Wilson-type transition for bulk divalent metals, ${ }^{36}$ many-body (electron-correlation) effects play a very important role in the nonmetal-tometal transition in both monovalent and divalent systems. From this point of view, a gradual nonmetal-tometal transition in divalent metal layers [as has been observed for $\mathrm{Hg} / \mathrm{Cu}(100)$, (Ref. 4) $\mathrm{Ba} / \mathrm{Ni}(111)$, (Ref. 4) $\mathrm{Hg} / \mathrm{W}$ (110), (Ref. 3) and also $\mathrm{Mg} / \mathrm{Mo}(112)$ (Refs. 6 and 7] is not unreasonable. This is particularly true since it has been concluded that the many-body effects are enhanced in low-dimensional systems. ${ }^{14,15,26,27}$

Applying Eq. (6) to the metallization of Mg overlayers, and to the experimental data of the electron effective 


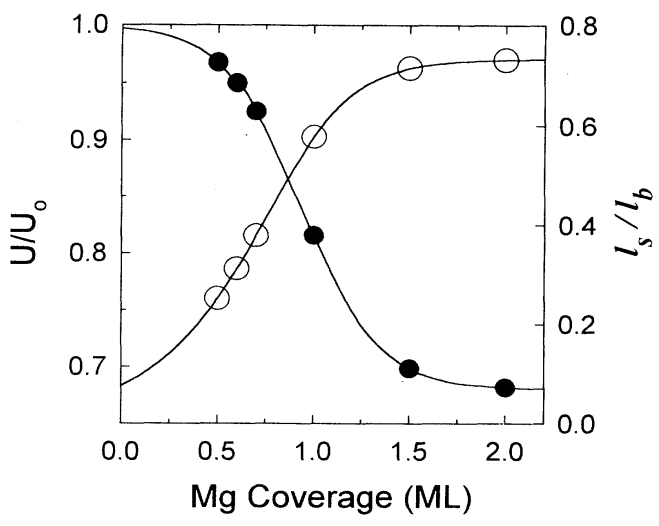

FIG. 6. The relative changes of the Coulomb repulsion $U(O)$ and the screening parameter $(O)$ (inverse of screening length) as function of $\mathrm{Mg}$ coverage on $\mathrm{Mo}(112) . U_{0}$ is assumed to be the electron-electron correlation at zero coverage and $l_{b}$ the screening parameter for bulk magnesium.

mass, a relative change of the Coulomb repulsion $U$ and the screening parameter $l_{s}$ (inverse of screening length) as function of coverage can be obtained. The results have been plotted out in the inset of Fig. 6. The validity of Eq. (7) and a general application of the Mott-Hubbard model to this system needs to be questioned, in spite of the apparent superficial agreement of the data to the theoretical work of Brinkman and co-workers. ${ }^{10-12}$ More theoretical study is needed if we are to understand how much of the Mott-Hubbard model can be legitimately applied to this and other divalent metal overlayer systems. The rel- ative changes in the Coulomb repulsion $U$ of Fig. 6 do not, in fact, resemble what is generally expected for a Mott-Hubbard system. Problems with the changes in the Coulomb repulsion energy are not confined solely to this system. Experiments with a system that exhibits a nonmetal-to-metal transition that should, in principle, more closely resemble a Mott-Hubbard system also can be seen to exhibit behavior that is difficult to explain. ${ }^{8}$ We can speculate about two possible sources of these problems: difficulties may be due to the dimensionality effects on the electron-electron correlation, or the origin of the difficulties may be due to the simplicity of the Hubbard model.

\section{CONCLUSION}

In summary, in divalent $\mathrm{Mg}$ overlayers a gradual nonmetal-metal transition is confirmed by the change in the $\mathrm{Mg}$ band dispersion and the electron effective mass with increasing $\mathbf{M g}$ coverage. An empirical relationship between the effective mass and the screening parameter across the nonmetal-to-metal transition has been obtained from angle-resolved and resonant photoemission experiments. This relationship resembles the scaling theory for the Mott-Hubbard transition surprisingly well.

\section{ACKNOWLEDGMENTS}

Helpful discussions with E. W. Plummer are gratefully acknowledged. This work was supported by NSF (No. DMR-92-21655 and No. DMR-94-96131). The experiments were carried out at the Synchrotron Radiation Center which is also funded by NSF.
${ }^{1}$ E. W. Plummer and P. A. Dowben, Prog. Surf. Sci. 42, 201 (1993), and references therein; E. W. Plummer, J. M. Carpinelli, H. H. Weitering, and P. A. Dowben, Phys. LowDimens. Struct. 4/5, 99 (1994), and references therein.

${ }^{2}$ C. Binns and C. Norris, J. Phys. Condens. Matter 3, 5425 (1991); C. Binns, C. Norris, and S. J. Gurman, J. Phys. C 16, 417 (1983).

${ }^{3}$ Jiandi Zhang, Dongqi Li, and P. A. Dowben, Phys. Lett. 173, 183 (1993); J. Phys. Condens. Matter 6, 33 (1994); J. Vac. Sci. Technol. A 12, 2190 (1994).

${ }^{4}$ P. A. Dowben, D. LaGraffe, Dongqi Li, G. Vidali, L. Zhang, L. Dottl, and M. Onellion, Phys. Rev. B 43, 10677 (1991).

${ }^{5}$ Dongqi Li, Jiandi Zhang, Sunwoo Lee, and P. A. Dowben, Phys. Rev. B 45, 11876 (1992).

${ }^{6}$ G. A. Katrich, V. V. Klimov, and I. N. Yakovkin, J. Electron. Spectrosc. Relat. Phenom. 68, 369 (1994); G. A. Katrich, V. V. Klimov, and I. N. Yakovkin, Ukranian J. Phys. 37, 429 (1992).

${ }^{7}$ Jiandi Zhang, D. N. McIlroy, and P. A. Dowben, Phys. Rev. B 49, 13780 (1994); Europhys. Lett. 29, 469 (1995).

${ }^{8}$ G. M. Watson, P. A. Brühwiler, H. J. Sagner, K. H. Frank, and E. W. Plummer, Phys. Rev. B 50, 17678 (1994).

${ }^{9}$ Nagindar Singh, P. A. D. M. A. Dale, D. Bullet, and R. G. Jones, Surf. Sci. 294, 333 (1993).

${ }^{10}$ W. F. Brinkman and T. M. Rice, Phys. Rev. B 2, 4302 (1970).
${ }^{11}$ N. H. March, M. Suzuki, and M. Parrinell, Phys. Rev. B 19, 2027 (1979); A. Ferraz, Phys. Lett. A 124, 442 (1987).

${ }^{12}$ D. Vollhardt, Rev. Mod. Phys. 56, 99 (1984).

${ }^{13}$ M. A. Continentino, Europhys. Lett. 9, 77 (1989); Phys. Rev. B 43, 6292 (1991); 45, 11312 (1992).

${ }^{14}$ Y.-R. Jang and B. I. Min, Phys. Rev. B 48, 1914 (1993).

${ }^{15}$ G. E. Santoro and G. F. Giuliani, Phys. Rev. B 39, 12818 (1989); S. Yarlagadda and G. F. Giuliani, Phys. Rev. B 49, 14188 (1994).

${ }^{16}$ R. Ramakumar, K. P. Jian, R. Kumar, and C. C. Chancey, Phys. Rev. B 50, 10122 (1994); R. Ramakumar, R. Kumar, K. P. Jian, and C. C. Chancey, ibid. 48, 6509 (1993).

${ }^{17}$ Y. Tokura, Y. Taguchi, Y. Okada, Y. Fujishima, T. Arima, K. Kumagai, and Y. Iye, Phys. Rev. Lett. 70, 2126 (1993); J. Phys. Chem. Solids 53, 1619 (1992); Y. Fujishima, Y. Tokura, T. Arima, and S. Uchida, Physica (Amsterdam) 185-189C, 1001 (1989); Y. Fujishima, Y. Tokura, T. Arima, and S. Uchida, Phys. Rev. B 46, 11167 (1992).

${ }^{18}$ O. M. Braun and V. K. Medvedev, Usp. Fiz. Nauk 157, 631 (1989) [Sov. Phys. Usp. 32, 328 (1989)]; V. K. Medvedev and I. N. Yakovkin, Fiz. Tverd. Tela. 21, 313 (1979); 23, 669 (1981) [Sov. Phys. Solid State 21, 187 (1979); 23, 397 (1981)].

${ }^{19}$ R. A. Bartynski, R. H. Gaylord, T. Gustafsson, and E. W. Plummer, Phys. Rev. B 33, 3644 (1986); U. O. Karlsson, G. V. Hansson, P. E. S. Persson, and S. A. Flodström, ibid. 26, 1852 
(1982).

${ }^{20}$ L. Ley, G. P. Kerker, and N. Mårtensson, Phys. Rev. B 23, 2710 (1981).

${ }^{21}$ J. C. Boettger and S. B. Trickey, J. Phys. Condens. Matter 1, 4323 (1989); E. Wimmer, J. Phys. F 14, 681 (1984).

${ }^{22}$ Wai-Ning Mei, Jiandi Zhang, D. N. McIlroy, P. A. Dowben, and M. Onellion (unpublished).

${ }^{23}$ G. D. Mahan, Many-Particle Physics, 2nd ed. (Plenum, New York, 1990), Chaps. 5 and 6, pp. 474-589.

${ }^{24}$ M. C. Gutzwiller, Phys. Rev. Lett. 10, 159 (1963); Phys. Rev. 137, A1726 (1965).

${ }^{25}$ X. G. Gong, Q. Q. Zheng, and Yi-Zhen He, Phys. Lett. A 181, 459 (1993).

${ }^{26}$ P. Hawrylak, Phys. Rev. Lett. 59, 485 (1987); J. E. Northrup, M. S. Hybertsen, and S. G. Louie, Phys. Rev. Lett. 59, 819 (1987).

${ }^{27}$ G. M. Watson, P. A. Bruhwiler, E. W. Plummer, H.-J. Sagner, and K.-H. Frank, Phys. Rev. Lett. 65, 468 (1990); E. Jensen and E. W. Plummer, ibid. 55, 1912 (1985); In-Whan Lyo and E. W. Plummer, ibid. 60, 1558 (1989).

${ }^{28}$ T. Adno, A. B. Fowler, and S. Stern, Rev. Mod. Phys. 54, 4 (1985).

${ }^{29}$ J. Lee, H. N. Spector, and P. Melman, J. Appl. Phys. 58, 1893 (1985).

${ }^{30}$ F. Stern and W. E. Howard, Phys. Rev. 163, 813 (1967); F. Stern, Phys. Rev. Lett. 18, 546 (1967).

${ }^{31}$ O. Hipolito and V. B. Campos, Phys. Rev. B 19, 599 (1981).

${ }^{32}$ P. Price, J. Vac. Sci. Technol. 19, 599 (1981).

${ }^{33}$ W. E. Edelstern and H. N. Spector, Surf. Sci. 224, 581 (1989).

${ }^{34}$ M. E. Garcia, G. M. Pastor, and K. H. Bennemann, Phys. Rev. Lett. 67, 1142 (1991); Z. Phys. D 19, 219 (1991).

${ }^{35}$ G. M. Pastor, P. Stampfli, and K. H. Bennemann, Europhys. Lett. 7, 419 (1988); C. Baladrón, M. E. Garcia, P. Stampfli, and K. H. Bennemann, Z. Phys. D 19, 215 (1991).

${ }^{36}$ N. F. Mott, Metal Insulator Transitions, 2nd ed. (Taylor and Francis, London, 1990). 